\title{
Realization of IEC 60870-5-104 Protocol in DTU
}

\author{
Weiqing Tao, Xiong Chen and Qiaoyun Zhang
}

\begin{abstract}
According to the IEC 60870-5-101 and IEC 60870-5-104 telecontrol protocol published by IEC, the corresponding companion standards, namely DL/T 634.5101-2002 and DL/T 634.5104-2002 have already been drafted in China. Application of IEC 60870-5-104 protocol in DTU is discussed detailedly in this paper, based on introducing the basic principles of the IEC 60870-5-104 protocol and DTU. The paper specifically describes Ethernet communication implementation based on hardware protocol stack Ethernet chip W5100 and DSP, which include hardware circuit design and software realization. Finally the paper introduce the software design of IEC 60870-5-104 protocol.
\end{abstract}

Index Terms-IEC 60870-5-104 protocol, DTU, Ethernet communication, W5100, DSP

\section{INTRODUCTION}

Switching station monitor terminal DTU is core device for switching station automation, which can remote monitoring the switch position, actuating signal of protection, the fault line select for the small current grounding, bus voltages, line currents and electrical measurement of the $10 \mathrm{KV}$ switching station in distribution network and remote control the switch[1-2]. Realization of switching station run data collect, switching station run state change and optimization of distribution network must through the communication system. Obviously, the communication system of the switching station automation system plays an important part in the distribution automation system, which is the nervous system of the distribution automation system. With the rapid development of communication technology and computer network technology, communication network of distribution automation system is also evolving. The internal communication of the early switching station usually uses serial communication (such as RS232/485). The advantage of serial communication is that the communication equipment is simple and low cost. The disadvantage is that the data can only be transmission by question-answer pattern and transmission efficiency is low. As the communication data of the switching station increase and the real-time of the communication demand higher, the communication mode based on ethernet technology become the inexorable trend for the development of switching station automation.

At the present time, the network transmission in china mainly uses IEC 60870-5-104, TASE 2 and DL/T476 transmission protocol. As an international standard protocol, IEC 60870-5-104 protocol has the advantage of good real-time, high reliability, large data traffic, easy to expand the amount of information and support network transmission[3]. The paper will introduce how to realize IEC 60870-5-104 protocol in DTU.

\section{ANALYSIS OF IEC 60870-5-104 PROTOCOL}

This standard defines the use of an open TCP/IP-interface to a network, containing for example a LAN for telecontrol equipment, which transports IEC 60870-5-101 ASDUs. Routers which include the different WAN-types (for example, X.25, Frame Relay, ISDN, etc.) may be connected via a common TCP/IP-LAN-interface (see figure 1). Figer1 shows a redundant configuration in the central station in addition to a non-redundant system.

The reference model IEC 60870-5-104 protocol used is from open interconnected ISO-OSI reference model. But, it uses only five layers and it is in the application layer protocol position [4-5]. There are many application layer protocols based on TCP/IP and each application layer protocol corresponds to a network port number. In the transport layer, IEC 60870-5-104 protocol use TCP protocol [6-7]. Its corresponding network port number is 2404 , and its structure show in figure 2.

As showed in figure 2, IEC 60870-5-104 protocol is actually the combination of IEC 60870-5-101 protocol and the network transmission function provided by TCP/IP, so IEC 60870-5-101 protocol can be used in a variety of network types within TCP/IP, such as X.25,FR,ATM and ISDN. 


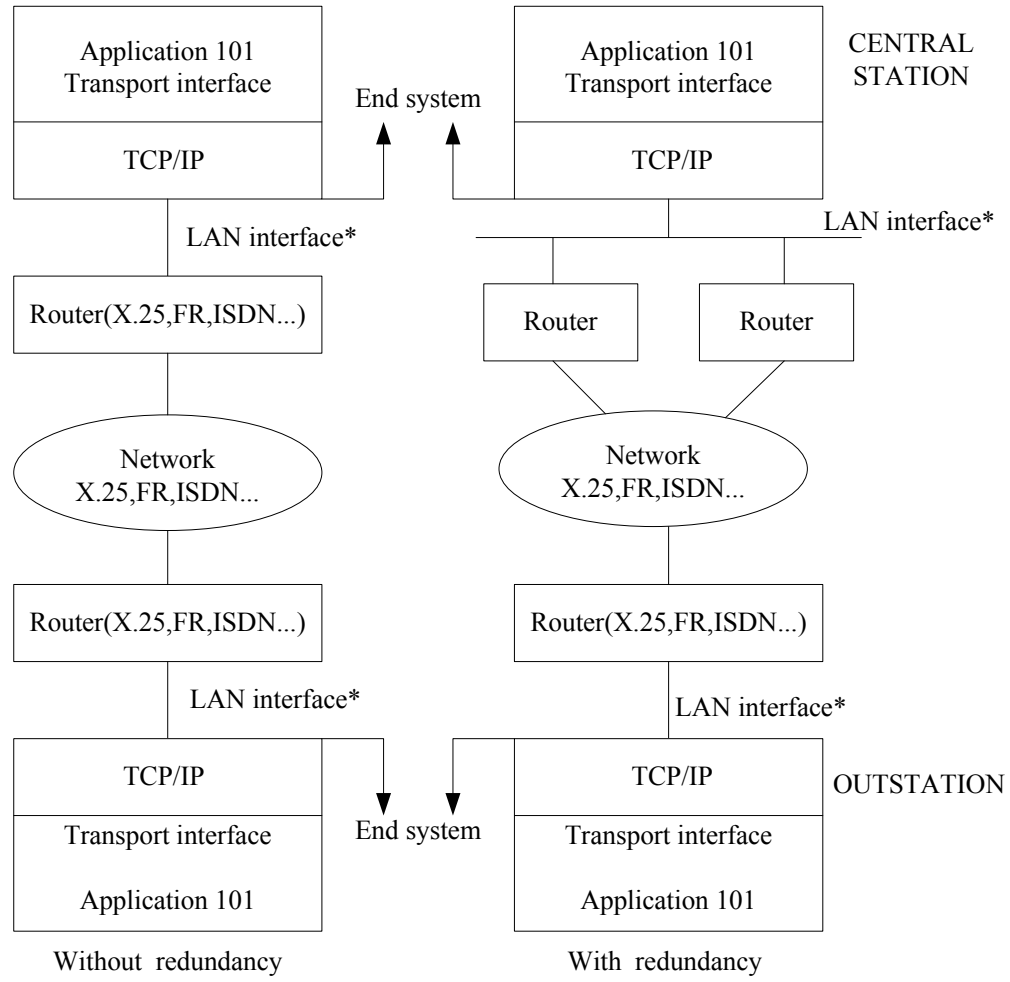

Fig.1 General Architecture

\begin{tabular}{|c|c|c|}
\hline $\begin{array}{c}\text { Selection of application functions of } \\
\text { IEC } 60870-5-5 \text { according to IEC } 60870-5-101\end{array}$ & Initialization & User process \\
\hline \multicolumn{2}{|c|}{ Selection of ASDU from IEC $60870-5-101$ and IEC $60870-5-104$} & \multirow[b]{2}{*}{ Application (layer 7) } \\
\hline $\begin{array}{l}\text { APCI(Application Protocol Control Informatio } \\
\text { Transport Interface(user to TCP interface) }\end{array}$ & & \\
\hline \multirow{4}{*}{\multicolumn{2}{|c|}{$\begin{array}{c}\text { Selection of } \\
\text { TCP/IP protocol suite(RFC 2200) }\end{array}$}} & Transport (layer 4) \\
\hline & & Network (layer 3) \\
\hline & & Link (layer 2) \\
\hline & & Physical (layer 1) \\
\hline
\end{tabular}

Fig.2 network reference model

IEC 60870-5-104 protocol provides the length of one APDU packet up to 255 bytes (include start character and length identification), so the maximum length of ASDU is 253. The length of APDU includes four octets of control field and ASDU, so the maximum length of ASDU is 249. This provision limits a APDU packet to send up to 121 normalized measured values without quality descriptor or 243 single-point information. If the number of information collected by the sub-station RTU or monitoring system exceed above limit, we have to divide one APDU into more to send. The structure of application protocol data unit is show as figure 3 .

The control field of APDU defines control information for the protection against loss and duplication of messages, start and stop of message transfers and the supervision of transport connections. It includes four octets and can be divided into three kinds of message format by its definition. Three types of control field formats are used to perform numbered information transfer (I format), numbered supervisory functions ( $\mathrm{S}$ format) and unnumbered control functions ( $\mathrm{U}$ format). 


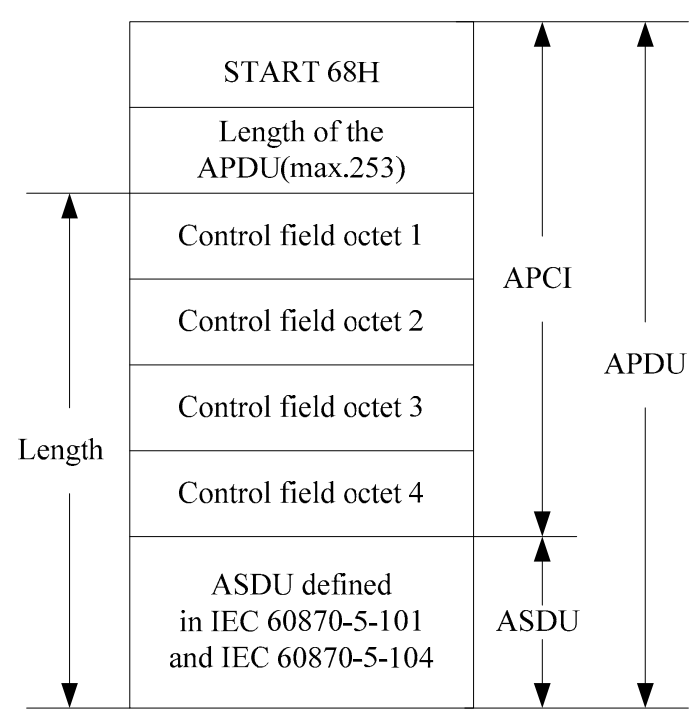

Fig. 3 APDU of the defined telecontrol companion standard

\section{SYSTEM HARDWARE CONFIGURATION}

Figure 4 shows the system diagram of DTU, it is a hierarchical distributed network architecture which uses separate modules interconnected through the network. The system is composed of communication manage machine, telemetry units, remote signaling units, remote control units, power modules, storage battery modules, display modules, communication interfaces etc. Communication manage machine, telemetry units, remote signaling units and remote control units of the system use stand-alone CPU.

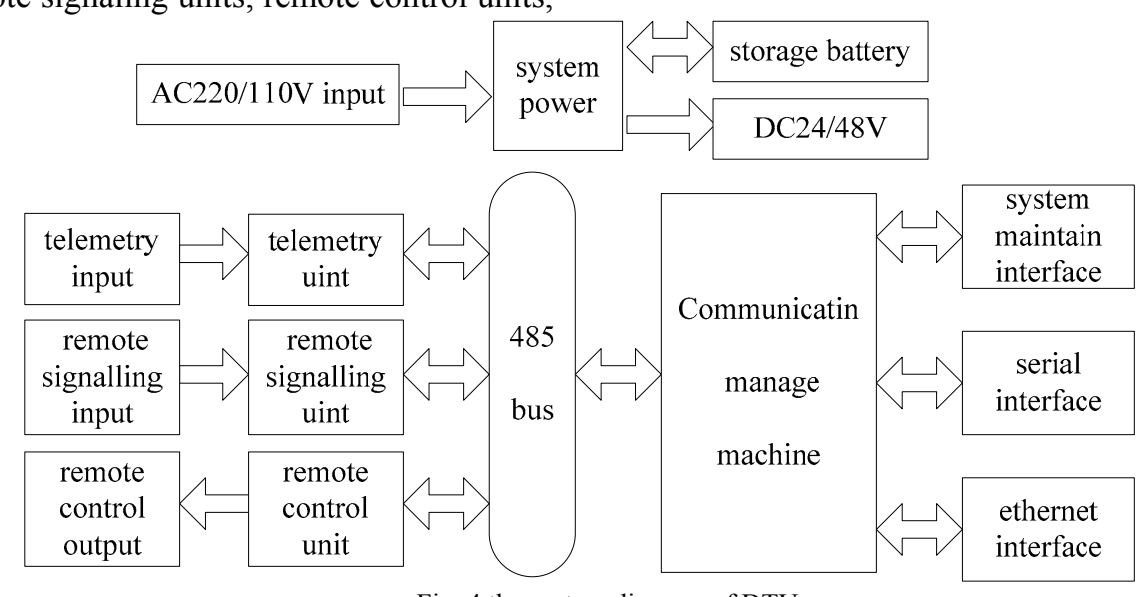

Fig. 4 the system diagram of DTU

As the core unit of whole system, Communication manage machine selects powerful 32-bit DSP TMS320F2812 as the core CPU. Communication manage machine up to communicate with the main station or distribution network sub-station through the GPRS/carrier or Ethernet, down to communicate with telemetry units, remote signaling units or remote control units though bus and can display running state and parameters of equipment by LCD. Telemetry unit consist of ultra-low-power microprocessor MSP430F5438 of TI Company and peripheral function modules. It uses six specific ADE7758 energy metering IC to collect multi-channel voltage and current of external transmission network. Remote control unit is designed based on 16-bit low-power high-performance microprocessor MSP430F5438, each unit control 16 relays and can read back the relay operating state. It is also responsible for battery activated treatment. Remote signaling unit collect quantity of state and record sequence of events based on microprocessor MSP430F5438. It can collect forty eight way switching value input and monitor battery status.

Communication manage machine equipped with one high-speed industrial Ethernet interface, as the interface of IEC 60870-5-104 communication protocol; two RS-232 (isolated) interface, one as communication interface for debugging and maintaining, one as the interface of IEC 60870-5-101 communication protocol; one RS-485 (isolated) interface, as the communication interface for communicating with various telemetry units, remote signaling units and remote control units.

\section{REALIZATION OF ETHERNET COMMUNICATION}

IEC 60870-5-104 protocol is a telecontrol protocol based on Ethernet TCP/IP protocol, so that we have to realize 
Ethernet communication function in order to provide physical interface for IEC 60870-5-104 protocol. The following text will describe in detail the realization of the Ethernet communication function.

\section{A .Realization scheme of Ethernet communication}

The current Ethernet solution scheme often use host CPU to connect the physical layer interface chip and program the Ethernet communication protocol in master controller. This method need complex programming and consumes a lot of time to debug. As the network protocols in general are relatively large and the stability of the network protocols software is poor, it is not conducive to rapid development and steady run for the system. And customers often have new demands. It means that it is difficult to meet the requirements for frequent update and upgrade. The paper use hardware protocol stack chip to realize Ethernet communication function of DSP. Hardware protocol stack chip select an internal hardware protocol stack network interface chip W5100 developed by WIZnet Company. We need not consider Ethernet control but just simple socket programming, which is as simple as an access to external memory. It provides two kinds of parallel bus interface (direct and indirect bus) and serial SPI interface. W5100 greatly reduces the workload of hardware interface design and network programming, and can realize a Reliable and stable operation of the remote data communication system. It can be widely used in a variety of security testing, measurement and monitoring of power system, audio and video transmission, remote information transmission. The paper will not describe W5100 in detail for lack of space, the detail description refer to the literature.

\section{B. Hardware circuit design}

There are three kinds of interface mode between W5100 and microprocessor chip: direct bus interface mode, indirect bus interface mode and SPI mode. Direct Bus Interface mode for transmission of large amounts of data; the interfacing of SPI mode is simple and it is suitable for small amount of data transmission and low transmission rate; Data transfer performance of indirect bus interface mode is in therange between them [8]. In this system, we use the direct bus interface mode, in order to maximize the data transmission rate.

Figure 5 show the interface circuit between F2812 and W5100 which use direct bus interface mode. Through connect the /CS pin of W5100 with the /XZCS2 pin of F2812, W5100 will be mapped to the ZONE2 area of F2812, which will occupy a total of $32 \mathrm{~K}$ address space from 0x80000 to 0x88000.The interface level of F2812 and $\mathrm{W} 5100$ are $3.3 \mathrm{~V}$, so we can connect them directly without level converter. GPIOB0 of F2812 as the reset signal of W5100, the read and write signal, address bus and data bus can connect directly between F2812 and W5100, the interrupt signal of W5100 connect to the F2812 XINT1 pin directly. It is easy to see, the design of hardware circuit is simple under this mode, which is similar to the RAM DSP extended and just increase reset and interrupt control signals. In terms of the F2812, the operation with the W5100 is just like reading and writing the RAM [9-10].

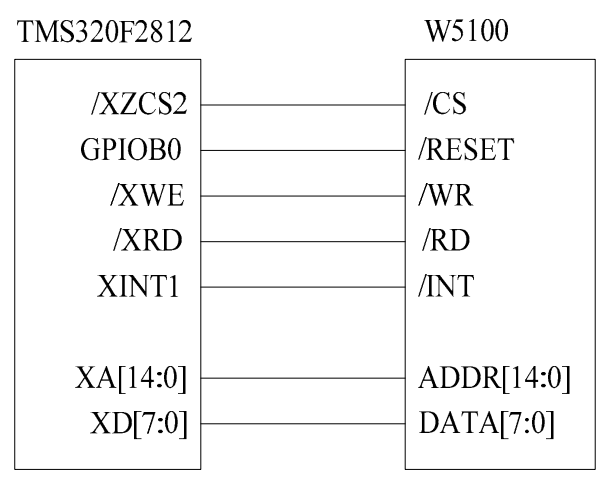

Fig.5 interface circuit between DSP and W5100

\section{Software design}

The software of Ethernet communication includes W5100 initialization, Socket initialization and Ethernet communication process establishment and data processing. WIZnet Company offers a complete Socket API Functions. It works similar to Socket API of Windows. It is very convenient to use $\mathrm{C}$ language to program in integrated development environment CCS of TI Company.

1) Initialization

W5100 initialization includes basic configuration initialization and network information set. Basic configurations mainly set mode register, interrupt mask register, retry time-value register and retry count register. Network information set mainly set gateway IP address register, source hardware address register, subnet mask register and source IP address register. Socket initialization is used to set Socket register information. Its main task is setting the port to TCP mode, checking the gateway and obtaining the physical address of the gateway and setting segment size.

\section{2) data communication}

There are two operating modes for application based on TCP, namely server mode and client mode. The difference between Server mode and client mode is that in the establishment of TCP connection, the server never initiate the connection request, it has been in a listening state, when listening to the connection from the client request, then accept this request, thereby establishing a TCP Connection. Server and client can send and receive data through the virtual communication link. IEC 60870-5-104 protocol defines that the controller (namely main station) is equivalent to the client (linker) and the slave station (namely sub-station) is equivalent to the server (listener) under normal circumstances. The DTU the paper designed is slave station, so it is set to server mode. Ethernet communication process in this mode show in Figure 6. The various processes of the communication flow chart are realized through Socket API function provided by WIZnet Company. The paper will take listen for example to illustrate for lack of space. It is necessary to set the port to listen mode when open the port. The API function used is unsigned char Socket_Listen (SOCKETs, unsigned int local Port), parameters indicate which port, and parameter localPort indicate port number. The port number in the paper is fixed to 2404 [11]. 


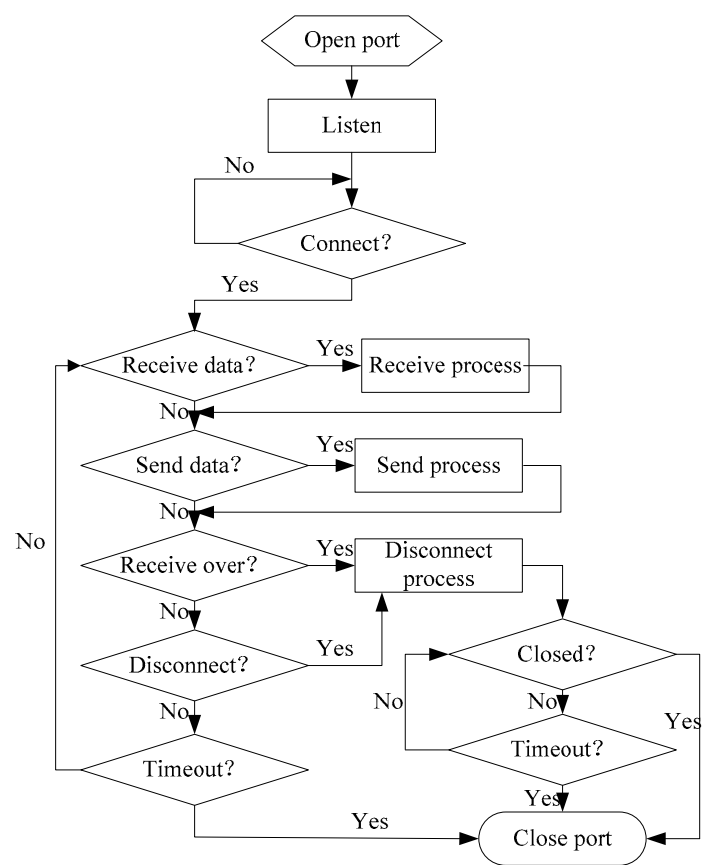

Fig.6 Ethernet communication process

\section{REALIZATION OF THE SOFTWARE OF IEC 60870-5-104 PROTOCOL}

In order to meet the needs of the power system development and satisfy the needs of different users, the paper not only realize the basic function of the DTU, but also realize the matching function according to the standard DLT721.It includes general interrogation, clock synchronization, telemetry, remote signal, remote control, wave recording, harmonic, parameter modification and watch distance and other functions. The paper uses balanced transmission. the design of the software of 104 protocol will be introduced as following.

\section{A. Data structure}

$\mathrm{U}$ format and $\mathrm{S}$ format do not need to define struct as the structure of their message are simple and fixed, reference to frame structure and ASDU format 104 protocol defined. So it only need to define struct for I format message. The struct consists of application data unit length, send sequence number, receive sequence number, type identification, variable structure qualifier, cause of transmission, common address of ASDU and information object address.

\section{B. Program structure}

Because there are only three frame format defined by 104 protocol, The program divide the soft module according to the frame format and send data according to the order of the frame format in strict, when it process the received data and the data send. The program structure makes it convenient to realize the program and also make the program structured, modular, easy to maintain.

\section{Program flow}

The following text will introduce the thread of entire communication program flow. The receive data process flow is showed in figure 7.

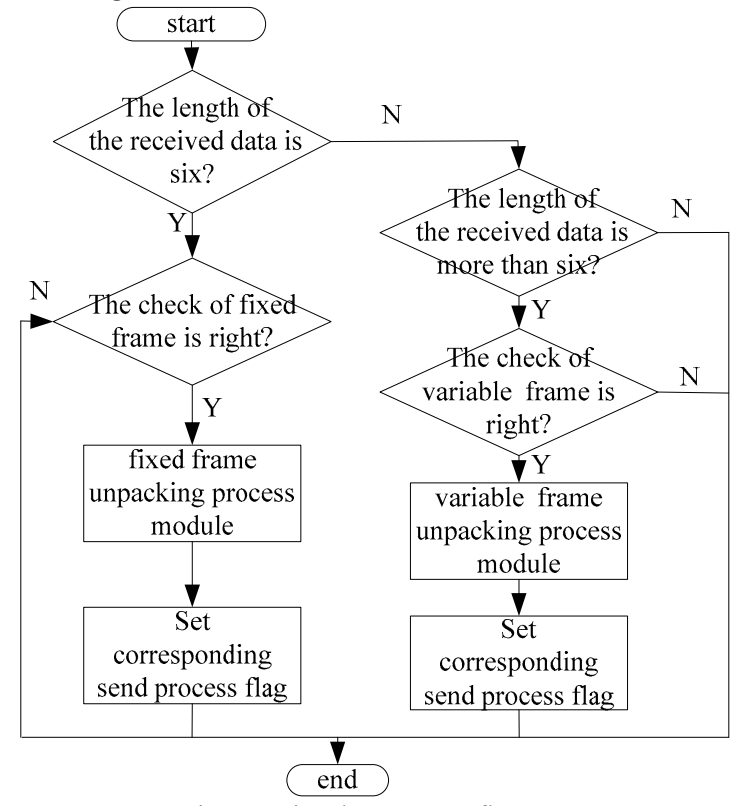

Fig. 7 receive data process flow 
When the Ethernet chip receives a packet, it will generate receive data interrupt. It will set the received data flag RecvFlag in the receive interrupt. The main function calls Ethernet handle function ProcessAboutNet ( ) once every ten milliseconds. The Ethernet handle function ProcessAboutNet ( ) will detect the flag RecvFlag, it will call receive data process function when RecvFlag is logical one. Receive data process function will read the received data from the receive data buffer of the W5100 and store it in memory, and then call the unpacking process functions. Unpacking process function distinguish the variable frame length message format(I format) from the fixed frame length message format( $U$ format or $S$ format) according to the frame length and then check the frame message according to its frame format. When the initial characters and the length are correct, the program enters the corresponding frame unpacking process module. It analysis the first octet of the control field and packing corresponding data according to the structure of the fixed frame length message in the fixed frame unpacking process module. It will set the send flag after packing. In the variable frame unpacking process module, it sets corresponding process flag according to the type identification of received data and calls send data process function. The send data process function packing corresponding data according to the process flag set in the variable frame unpacking process module. It will also set the send flag after packing. In the Ethernet process function, it will detect the send flag and will send the packed data through Ethernet chip W5100 when the send flag is logical one Till then, a complete sending and receiving data processing is over.

\section{CONCLUSION}

As IEC 60870-5-104 protocol is applied in practical engineering, it shows that china move closer to international standards in dispatching automation. It changes the present situation that the power network dispatching automation system transfers real-time data only through serial communication mechanism. The paper describes the realization of 104 protocols in DTU. It includes the realization of Ethernet communication function of DTU based on hardware protocol stack chip and software design of 104 protocols. It has greatly increased the communication efficiency and real-time of DTU and can meet the requirement of communication data quantity for the expansion of DTU. In addition, the adoption of 104 protocols meet the requirements of power network technology development and have positive significance for promoting power network automation and ensuring safe operation of power network.

\section{REFERENCES}

[1] Yongjun Lin,Yujie Shi.Distribution Automation Practical Technology. China Water Conservancy and Hydropower Press:Bei Jing,2008,pp.52-68.

[2] Jing Gong. Distribution Integrated Automation Technology.China Machine Press: Bei Jing, 2008, pp. 67-73.

[3] Qiang Li,Yongli Zhu. Applications of IEC 60870-5-104 protocol in substation automation system.Telecommunications for Electric Power System,2007,28(182).

[4] IEC60870-5-104, Telecontrol Equipment and Systems (Part 5-104: Transmission Protocols, Network Access for IEC 60870-5-101 Using Standard Transport Profiles) [S].

[5] Yang Ju,Huigang Zhang. Design and application of IEC 60870-5-104 telecontrol protocol, 2006, 34 (17).

[6] Yuan Zhao, Zhijian Shen. Application of TCP/IP based IEC 60870-5-104 telecontrol protocol in power system.2003, 27 (10).

[7] Long Du,Luning Shi,Jinbai Yang.Application of IEC 60870-5-104 telecontrol protocol based on TCP/IP in direct dispatching station.2008, 36 (17).

[8] WIZNET Corporation.W5100 Datasheet (version 1.1.6).2008

[9] Yiyi Zou, Canxin Guo etc. Design of DSP Embedded System Based on Ethernet Hardware Protocol Stack.2008, 29 (11).

[10] Zibin He,Fajie Duan etc. DSP-based Network Embedded Vision System.2008, (2).

[11] Yiyi Zou, Canxin Guo etc. Application of W5100 in DSP Remote Ethernet Communication System.Industrial Control Computer, 2008, $218)$.

Weiqing Tao is a Member of IEEE, is working as an Assistant Professor in the College of Electrical Engineering and Automation in Hefei University of technology,. Anhui, China. He has more than 50 publications in National / International conferences and Journals. His areas of interest include Distribution Automation, Communication, Artificial Intelligence and his Emailiswqtao@mail.hf.ah.cn.

Xiong Chen, is a graduate student in the College of Electrical Engineering and Automation in Hefei University of technology,.

Qiaoyun Zhang is a graduate student in the College of Electrical Engineering and Automation in Hefei University of technology. 